THU0352

ASSOCIATION BETWEEN MEMORY B-CELLS AND PHENOTYPIC FEATURES OF SJÖGREN'S SYNDROME

${ }^{1,2,3}{ }^{2}$. Barcelos, C. Martins ${ }^{1}$, A.L. Papoila ${ }^{4}$, C. Geraldes ${ }^{4}$, J. Cardigos $^{5}$, G. Nunes ${ }^{1}$, T. Lopes ${ }^{1}$, N. Alves ${ }^{5}$, J. Vaz-Patto ${ }^{2}$, J.C. Branco ${ }^{1,3}$, L.M. Borrego ${ }^{1} .{ }^{1}$ CEDOC, Nova Medical School; ${ }^{2}$ Rheumatology, Instituto Português de Reumatologia;

${ }^{3}$ Rheumatology, Hospital Cuf Descobertas; ${ }^{4}$ Nova Medical School; ${ }^{5}$ Ophthalmology, CHLC, Hospital de Santo António dos Capuchos, Lisbon, Portugal

Background: B-cell disturbances are a hallmark of pSS and play a pivotal role in the disease pathogenesis and clinical evolution, and may as well have a potential role in diagnosis. In pSS, an increase of the naïve subset and a decrease of memory B-cells have been reported. A decreased frequency of memory cells has also been identified in patients with Sicca syndrome without criteria for pSS

Objectives: Our study aims to evaluate the distribution of B-lymphocyte subpopulations in pSS and Sicca patients and to establish cut-off points for pSS classification in relation to healthy controls. Moreover, we aim to evaluate the relation between lymphocyte subpopulations and phenotypic features in pSS.

Methods: Fifty-seven pSS patients, 68 non-Sjögren Sicca patients and 24 healthy controls were included. Circulating B-cell frequencies were determined by flow cytometry, and the naïve and memory (switched and unswitched) subsets were characterised based on surface marker expression of the following monoclonal antibodies: CD19, CD24, CD27, Anti-IgD and Anti-IgM.

Kruskal-Wallis test was applied for groups' comparison. ROC curves were used to establish cut-off points in the B-cells subset levels and to estimate corresponding sensitivity and specificity. Data analysis was performed with $R$ software.

Results: Absolute numbers of lymphocytes in pSS were lower compared to controls, with Sicca presenting intermediate levels.

Significant differences were found between pSS and controls in absolute counts of all memory populations: total memory (TMem) $\left(\mathrm{CD} 19^{+} \mathrm{CD} 27^{+}\right)$, switched (SwM) $\left(\mathrm{CD} 19^{+} \operatorname{lgD}{ }^{-} \mathrm{CD} 27^{+}\right)$and unswitched memory (UnSwM) $(\mathrm{p}<0.001$ for all). Comparing pSS with controls, we found lower percentages of TMem in patients $(p=0.078$ and more significant differences in the UnSwM subset $(p=0.043)$. Percentages of memory B-cells in Sicca were not significantly different from pSS and controls. Absolute memory B-cells numbers in Sicca were intermediate between those of pSS and controls.

Through ROC curves, the B-cell subsets that better discriminate between pSS and controls were TMem and SwM. A cut-off of equal to $58 \mathrm{TMem}$ cells/ $\mathrm{\mu l}$ yelded a specificity of 0.88 and a sensitivity of 0.60 for pSS, and was met by $59.6 \%$ of pSS, $12.5 \%$ of controls and $38.8 \%$ of Sicca, and a cut-off of equal to 23.5 SwM cells/ $\mu$ lyelded a specificity of 0.88 and a sensitivity of 0.54 and was met by $54.4 \%$ of pSS, $12.5 \%$ of controls and $37.3 \%$ of Sicca.

pSS patients with lower values than the established cut-off points had longer disease duration, higher disease activity (ESSDAI), and were more likely to present auto-antibodies and positive biopsy. Several Sicca patients also presented memory B-cell subsets counts lower than the pSS cut-off, but no consistent differences in clinical profile were identified.

Conclusions: Decreased numbers of memory B-cell subsets clearly discriminate pSS from healthy controls. Lower memory B-cells counts are associated with more active pSS disease profile. It remains to be clarified whether Sicca patients with decreased memory B-cells represent pSS and if B-cell profiling could help in the diagnosis of pSS.

Disclosure of Interest: None declared

DOI: 10.1136/annrheumdis-2018-eular.5717

\section{THU0353 LUNG ULTRASOUND OF PLEURAL IRREGULARITIES (PI-US) IN PRIMARY SJÖGREN'S SYNDROME (PSS)- ASSOCIATED INTERSTITIAL LUNG DISEASE(ILD): CLINICAL, FUNCTIONAL, RADIOGRAPHIC AND ULTRASONOGRAPHIC SHORT-TERM FOLLOW-UP}

${ }^{1}$ F. Ferro, A. Bulleri ${ }^{2}$, A. Delle Sedie ${ }^{1}$, S. Barsotti ${ }^{1}$, N. Luciano ${ }^{1}$, E. Elefante ${ }^{1}$, M. Mosca ${ }^{1}$, C. Baldini ${ }^{1} .{ }^{1}$ Rheumatology Unit, University of Pisa; ${ }^{2}$ Radiology Unit, University of Pisa, Pisa, Italy

Background: Ultrasound of Pleural Irregularities (PI-US) has recently been suggested as a useful tool for the diagnosis and the assessment of interstitial lung disease (ILD) in primary Sjögren's Syndrome (pSS). However, no data are available regarding its role in the post-therapy evaluation of pSS-ILD.

Objectives: Aim of this study was to describe the post-therapeutic changes of the pleural profile in a monocentric cohort of pSS-ILD patients analysing clinical, functional, US and radiographic features.

Methods: Eighteen patients with pSS-ILD were included in the study. PI-US, HRCT and pulmonary function test (PFT) were performed in each patient at baseline. In 15/18 patients who had been treated according to international guidelines for ILD, clinical assessment, PFT and PI-US were repeated after 6 months. PI-US was performed by a single operator using a MyLab-25 (Esaote), $10 \mathrm{MHz}, 5 \mathrm{~cm}$ linear probe. PI was defined as the loss of normal hyperechoic linear pleural contour (score 0-2: normal, minimal and major changes at each intercostal space). PI-US total score represented the sum of partial scores assigned to 6 lung fields ( $2 \mathrm{for}$ the anterior, 2 for postero-superior and 2 for postero-inferior chest surface). HRCT- abnormal findings, distinguishing C-NSIP, F-NSIP and UIP pattern, were quantified using the Warrick score system

Results: Eighteen pSS-ILD patients ( $14 \mathrm{~F}: 4 \mathrm{M}$, mean age $=68.8 \pm 9.9$ years) were included in the study. The median PI-US score was 45 (range 25.5-73.5). Both $\mathrm{PI}$-US total score and partial postero-inferior PI-US score strongly correlated with the Warrick HRCT score $(r=0.813, p=0.000$ and $r=0.914, p=0.000)$ and inversely correlated with FVC $(r=-0.849, p=0.000$ and $r=-0.836, p=0.000)$, TLC $(r=-0.895$ $\mathrm{p}=0.000$ and $\mathrm{r}=-0.829, \mathrm{p}=0.000)$, and DLCO $(\mathrm{r}=-0.953, \mathrm{p}=0.000$ and $\mathrm{r}=-0.883$, $\mathrm{p}=0.001)$. Both PI-US score and PI-US of the poster-inferior field directly corre lated with FEV1/SVC $(r=0.701, p=0.004$ and $r=0.619, p=0.01)$ and with FEV1/ FVC $(r=0.600, p=0.02$ and $r=0.501, p=0.05)$. After 6 months of therapy, 15 patients (12F:3M, mean age 65.3 years) presenting at HRCT different ILD pattern distributions (4 C-NSIP, 7 F-NSIP, 4 UIP), were re-evaluated after appropriate medical treatment. Out of these 15 patients, 5 had been treated with glucocorticoids (GC) alone, 2 with azathioprine, 4 with hydroxychlorochine, 2 with Mycophenolate Mofetil, 1 with Rituximab and 1 with abatacept. Clinical, radiographic and functional evaluation showed stability of all parameters in the majority of the patients (13/15), maintaining unchanged the correlations with PI-US scores Moreover, 2/15 patients with active C-NSIP pattern, showed a significant improvement in clinical, radiographic and functional parameters. In these patients a significant reduction of the PI-US scores was also observed (PI-US score from 22 to 10 and from 57 to 30 ).

Conclusions: This study confirms the usefulness of PI-US evaluation for the diagnosis and assessment of pSS-ILD, demonstrating a strong correlation between PI-US and both HRCT findings and PFT. Furthermore, the significant reduction of $\mathrm{PI}$-US score in patients with clinical, radiographic and functiona improvement suggests that this tool may play a role in the follow-up of treated patients with active pSS-ILD.

Disclosure of Interest: None declared

DOI: 10.1136/annrheumdis-2018-eular.5955

\section{THU0354 IMPACT OF BIOLOGIC THERAPY IN SJOGREN'S SYNDROMEPATIENTS WITH OVERLAPPING AUTOIMMUNE DISEASES OR EXTRAGLANDULAR MANIFESTATIONS. A SYSTEMATIC REVIEW OF LITERATURE}

${ }^{1}$ G.E. Fragoulis, E. Nikiphorou ${ }^{2}$, A. Alunno ${ }^{3}, \mathrm{~F} . \mathrm{Ng}^{4}$, A.G. Tzioufas ${ }^{5} .{ }^{1}$ Institute of Infection, Immunity and Inflammation, University of Glasgow, Glasgow; ${ }^{2}$ Academic Rheumatology Department, King's College London, London, UK; ${ }^{3}$ Rheumatology Unit, University of Perugia, Perugia, Italy, ${ }^{4}$ Institute of Cellular Medicine, University of Newcastle, Newcastle, UK; ${ }^{5}$ Pathophysiology Department, University of Athens, Athens, Greece

Background: Treatment of Sjogren's syndrome (SS) has traditionally focused on conventional synthetic DMARDs (csDMARDs), with encouraging evidence on the benefit of biologic therapies emerging, mainly for treatment of SS extraglandular manifestations. Overlapping autoimmune diseases in SS are poorly studied; yet evidence primarily from case reports suggest a beneficial effect with biologics. Objectives: To systematically review the literature on the treatment of SS with biologics, taking a focus on case reports and overlapping autoimmune conditions or extraglandular manifestations (defined here as those described in the EULAR SS disease activity index - ESSDAI)

Methods: A literature review was performed independently by two reviewers, using Pubmed and the following search terms: "Sjogren" or "Sjogren's" AND any of the following: "biologics", "Etanercept", "Adalimumab", "Infliximab", "Golimumab", "Certolizumab", "Tocilizumab", "Abatacept", "Rituximab", "Belimumab", "Secukinumab", "Ustekinumab" and "Anakinra". Inclusion criteria were: articles in English; published until January 2018; case reports of patients with primary or secondary SS. Initial screening was based on title/abstract, followed by full-text review for articles fulfilling inclusion criteria. For articles written in a different language, information was obtained from abstract if available, otherwise excluded Concordance in article screening was $95 \%$ across the two reviewers. Data extraction focused on reporting overlapping autoimmune diseases and extraglandular manifestations, treatment and response data.

Results: Out of 679 papers screened, 39 articles were included. 22 overlapping autoimmune conditions were reported in 22 SS patients (table 1). Most of the patients were treated with Rituximab $(63.6 \%)$, while TNF-inhibitors $(22.7 \%)$, Tocilizumab (9.1\%) and Ustekinumab (4.5\%) were also used. Concurrent treatment with csDMARDs and steroids was used in $28.6 \%$ and $42.9 \%$ of the cases, respectively. $61.9 \%$ and $13.6 \%$ of the patients were csDMARDs- and biologic- experienced, respectively. Good response of overlapping condition was seen in $86.4 \%$ of them, while in $13.6 \%$, control or partial response was reported. Although, most of the studies do not mention the effect of biologic treatment on SS, genera 
'improvement' was mentioned in 4 cases, while arthritis was improved in 6 patients, 4 of which had secondary SS.

In terms of extraglandular manifestations (e.g cryoglobulinemic vasculitis, interstitial nephritis) 18 were reported. 16 (88.8\%) patients received Rituximab (one of them in combination with Belimumab), while $2(11.1 \%)$ were treated with TNFinhibitors. $11.1 \%$ and $61.1 \%$ of them received concurrent treatment with csDMARDS and steroids, respectively. $55.5 \%$ and $11.1 \%$ of the patients were csDMARDs- and biologics- experienced, respectively. Extraglandular manifestations responded well in the majority $(83.3 \%)$ of the patients, with the remaining having partial or late response. SS and arthritis 'improvement' was mentioned in 5 and 1 patients, respectively.

Table 1 Biologic treatments used for overlapping autoimmune conditions in SS patients. csDMARDs: conventional synthetic Disease Modifying Antirheumatic Drugs, NA: Not Applicable, Joints: improvement of arthritis. ^SS response was defined as clinical improvement of sicca symptomatology or improvement in ESSDAI. Improvement of arthritis was reported separately. $\uparrow$ cases of secondary Sjogren's syndrome ( 3 cases co-existing with RA and one case with SLE)

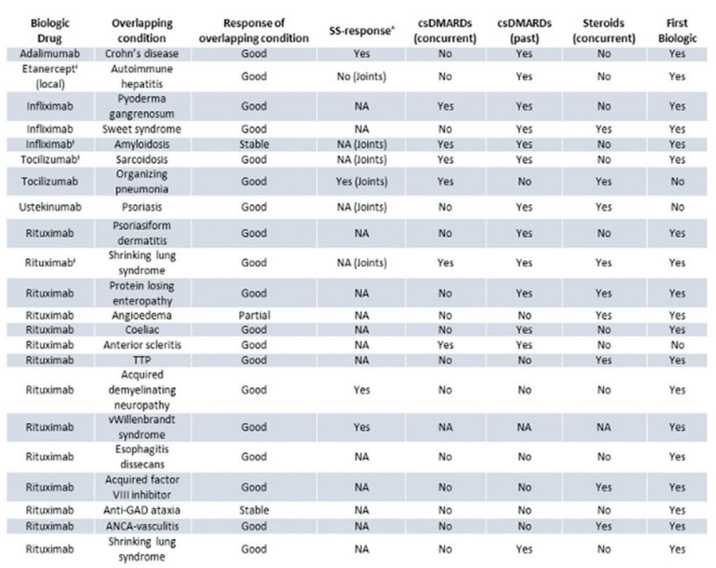

Conclusions: Treatment with biologic DMARDs, sometimes accompanied by steroids, appears to be beneficial also in treating overlapping autoimmune diseases as well as some extraglandular manifestations in SS patients.

Disclosure of Interest: None declared

DOI: 10.1136/annrheumdis-2018-eular.7200

\section{THU0355 DAMAGE ACCRUAL AND MORTALITY RATES AMONG DIFFERENT AGE GROUPS IN A COHORT OF PATIENTS WITH LUPUS}

G.A. Ramirez, B. Tejera-Segura, R. Lopez, A. Rahman, D.A. Isenberg. Centre for Rheumatology, University College London (UCL) Hospital NHS Foundation Trust, London, UK, London, UK

Background: Systemic lupus erythematosus (SLE) is a chronic, multi-system autoimmune disease, characterised by relapses and remissions, which eventually can lead to progressive, irreversible organ damage accrual. SLE-related damage implies increased morbidity and impaired quality of life. In addition, it constitutes itself a risk factor for the development of further damage and associates with early mortality. Age at disease onset has been postulated to affect the presenting clinical phenotype, the rate and likelihood of accruing damage and eventually mortality risk. Early-onset SLE patients have been reported to present with a more aggressive disease, especially in the first years after diagnosis. However, longterm data from large cohorts are scanty.

Objectives: To assess retrospectively the mortality and damage accrual rates in a large cohort of patients with SLE.

Methods: Demographic, clinical and laboratory data from 692 consecutive patients with SLE (631 females, 61 males), diagnosed according to the revised American College of Rheumatology (ACR) criteria in a single tertiary referral centre, were retrospectively analysed focusing on age of diagnosis. Damage accrual was assessed according to the Systemic Lupus Erythematosus
International Collaborating Clinics (SLICC)/ACR damage index (SDI). Cox's regression analysis, chi-square test, Kruskal-Wallis test and ANOVA were employed as appropriate.

Results: Thirty-three out of 692 patients were diagnosed with SLE before the age of 12,172 between 12 and 20, 443 between 21 and 50 and 44 after 50 years of age. As previously reported, a female preponderance was more evident in the central part of the age spectrum $(p=0.015)$. Nephritis and decreased complement were more frequent in patients with early-onset SLE $(p=0.001$ and $p=0.033$ respectively), serositis in the central age groups ( $p=0.025)$, and arthritis in lateonset patients $(p=0.002)$. Neuropsychiatric manifestations were less frequent in patients aged $>50$ years $(p=0.013$; table 1$)$. The global incidence rate for any damage was 48.36 per 1000 persons-years, whereas the death incidence rate was 8.54 per 1000 persons-years. Late-onset SLE associated with a higher risk of damage accrual $(H R=1.63, p=0.024)$ and of death $(H R=6.22, p<0.001)$. However there were no significant differences in the time to first damage, to death afte diagnosis and to death after the development of the first damage item, according to the age of diagnosis.

Conclusions: Younger patients with SLE show a distinct clinical phenotype, but share an accelerated accrual of morbidity and a higher risk of early mortality with patients of older age. Identifying age-specific predictors of disease severity will be of outstanding importance to improve long-term survival rates and patients' quality of life.

\section{REFERENCES}

[1] Lopez R, et al. Rheumatology 2012.

[2] Ambrose N, et al. Lupus 2016.

[3] Lim LSH, et al. Arthritis Care Res, 2017.

Disclosure of Interest: None declared

DOI: 10.1136/annrheumdis-2018-eular.3896

\section{THU0356 SYNERGISTIC EFFECT OF CUMULATIVE CORTICOSTEROID DOSE AND IMMUNOSUPPRESSANTS ON AVASCULAR NECROSIS IN PATIENTS WITH SYSTEMIC LUPUS ERYTHEMATOSUS}

${ }^{1}$ H.-H. Kwon, S.-Y. Bang ${ }^{1}$, S. Won ${ }^{2}$, Y. Park ${ }^{2}$, J.-H. Yi ${ }^{3}$, Y.B. Joo ${ }^{1}$, H.-S. Lee ${ }^{1}$, S. C. Bae ${ }^{1} .{ }^{1}$ Department of Rheumatology, Hanyang University Hospital For Rheumatic Diseases; ${ }^{2}$ Clinical Research Center for Rheumatoid Arthritis (CRCRA), Seoul; ${ }^{3}$ Department of Internal medicine, Hanyang University Guri Hospital, Guri, Korea, Republic of Ireland

Background: Avascular necrosis (AVN) is one of the most common organ damage in patients with systemic lupus erythematosus (SLE) and often causes serious physical disability.

Objectives: The aims of this study were to investigate clinical risk factors associated with symptomatic AVN and to analyse their synergistic effects in a large SLE cohort in Korea.

Methods: Patients with SLE were enrolled and followed from 1998 to 2014 in the Hanyang BAE Lupus cohort, in whom damage was measured annually according to the Systemic Lupus International Collaborating Clinics/American College of Rheumatology Damage Index. AVN was confirmed by imaging study if patients had symptoms. To determine risk factors for AVN, clinical, laboratory, and therapeutic variables were analysed by logistic regression. Relative excess risk due to interaction (RERI), attributable proportion (AP), and synergy index (S) were calculated to measure interactions between significant variables.

Results: Among 1,219 SLE patients, symptomatic AVN was the most common type of musculoskeletal damage $(10.8 \%, n=132)$. SLE patients with AVN showed an earlier onset age, demonstrated AVN more commonly in conjunction with certain other clinical manifestations such as renal and neuropsychiatric disorders, and received significantly higher total cumulative corticosteroid dose and immunosuppressive agents than did patients without AVN. However in multivariable analysis, only two variables including use of a cumulative corticosteroid dose greater than $20 \mathrm{~g}$ (odds ratio $(\mathrm{OR}) 3.08, \mathrm{p}=0.005$ ) and use of immunosuppressants including cyclophosphamide or mycophenolate mofetil (OR 4.34, $\mathrm{p}=0.002$ ) remained as significant risk factors for AVN. Patients with cumulative corticosteroid dose $>20 \mathrm{~g}$ and immunosuppressants use had

\begin{tabular}{|c|c|c|c|c|c|c|c|c|c|c|c|}
\hline $\begin{array}{l}\text { Age group } \\
\text { (years) }\end{array}$ & $\mathrm{N}$ & $\begin{array}{c}\text { Females } \\
(\%)\end{array}$ & $\begin{array}{c}\text { Nephritis } \\
(\%)\end{array}$ & $\begin{array}{c}\text { Neurological inv. } \\
(\%)\end{array}$ & $\begin{array}{c}\text { Arthritis } \\
(\%)\end{array}$ & $\begin{array}{l}\text { Rash } \\
(\%)\end{array}$ & $\begin{array}{c}\text { Photosensitivity } \\
(\%)\end{array}$ & $\begin{array}{c}\text { Oral ulcers } \\
(\%)\end{array}$ & $\begin{array}{c}\text { Serositis } \\
(\%)\end{array}$ & $\begin{array}{c}\text { antiDNA } \\
(\%)\end{array}$ & $\begin{array}{c}\text { Reduced complement } \\
(\%)\end{array}$ \\
\hline$<12$ & 33 & 78.8 & 48.5 & 27.3 & 72.7 & 81.8 & 42.4 & 27.3 & 18.2 & 72.7 & 51.5 \\
\hline $12-20$ & 172 & 89.5 & 41.9 & 22.1 & 87.2 & 65.7 & 39.0 & 30.2 & 37.8 & 66.9 & 55.0 \\
\hline $21-50$ & 443 & 93.2 & 33.4 & 22.6 & 91.9 & 66.8 & 41.8 & 26.0 & 41.1 & 61.2 & 46.0 \\
\hline$>50$ & 44 & 86.4 & 11.4 & 6.8 & 93.2 & 54.5 & 27.3 & 22.7 & 27.3 & 63.6 & 31.8 \\
\hline
\end{tabular}

